\title{
Structural Insights into the Altering Function of CRMP2 by Phosphorylation
}

\author{
Takuya Sumi ${ }^{1}$, Tsuyoshi Imasaki ${ }^{1,2,3}$, Mari Aoki $^{2}$, Naoki Sakai ${ }^{2}$, Eriko Nitta ${ }^{1}$, Mikako Shirouzu$^{2}$, and \\ Ryo Nitta ${ }^{1,2 *}$ \\ ${ }^{1}$ Division of Structural Medicine and Anatomy, Kobe University Graduate School of Medicine, Kobe, Hyogo \\ 650-0017, Japan, ${ }^{2}$ RIKEN Center for Life Science Technologies, Tsurumi-ku, Yokohama 230-0045, Japan, \\ ${ }^{3}$ JST, PRESTO, 4-1-8 Honcho, Kawaguchi, Saitama 332-0012, Japan
}

\begin{abstract}
Collapsin response mediator protein 2 (CRMP2) regulates neuronal polarity by controlling microtubule dynamics. CRMP2 activity is regulated by semaphorin-induced phosphorylation at the C-terminal tail domain. Unphosphorylated CRMP2 induces effective axonal microtubule formation to give the axonal characteristics to a neurite, whereas phosphorylated CRMP2 leads to the apparently opposite effect, growth cone collapse. We have recently characterized the structural detail of CRMP2-induced axonal microtubule formation (Niwa et al. (2017) Sci. Rep., 7: 10681). CRMP2 forms the hetero-trimer with GTP-tubulin to induce effective axonal microtubule formation in the future axon. Phosphorylation of CRMP2 has been reported to decrease the affinity between CRMP2 and the microtubule, albeit the molecular mechanisms of how the phosphorylation of CRMP2 changes the structure to achieve distinct effects from unphosphorylated CRMP2 is not well understood. Here we performed a series of biochemical and structural analyses of phospho-mimic CRMP2. Phosphorylation of CRMP2 undergoes small conformational changes at the C-terminal tail with shifting the surface charge, which not only alters the interactions within the CRMP2 tetramer but also alters the interactions with GTP-tubulin. Consequently, phospho-mimic CRMP2 fails to form a hetero-trimer with GTP-tubulin, thus losing the ability to establish and maintain the axonal microtubules.
\end{abstract}

Key words: CRMP2, phosphorylation, microtubule, axon, crystal structure

\section{Introduction}

A microtubule associated protein collapsing response mediator protein 2 (CRMP2/UNC33/Ulip2/CRMP-62/TOAD-64/ DRP-2) is necessary for establishing neuronal polarity through specification and elongation of the axon (Arimura and Kaibuchi, 2007; Arimura et al., 2004). Vertebrate CRMP2 gives the axonal characteristics to cultured hippocampal neurons by direct binding of CRMP2 to tubulin hetero-dimers to induce effective axonal microtubule formation (Fukata et al., 2002a, 2002b; Inagaki et al., 2001; Niwa et al., 2017). CRMP2-induced axonal microtubules are also used by molecular motor kinesin-1 for polarized transport in neurons (Maniar et al., 2012; Nakata et al., 2011; Niwa et al., 2017). However, the founding CRMP protein, UNC-33, was originally identified as a signaling molecule of semaphorin $3 \mathrm{~A}$ to induce the apparently opposite effect, growth cone collapse (Goshima et al., 1995; Li

\footnotetext{
*To whom correspondence should be addressed: Ryo Nitta, Division of Structural Medicine and Anatomy, Kobe University Graduate School of Medicine, Kobe, Hyogo 650-0017, Japan.

Tel: +81-78-382-5320

E-mail: ryonitta@med.kobe-u.ac.jp
}

et al., 1992; Maniar et al., 2012), albeit later studies resolved the contradiction.

CRMP2 is a downstream component of the semaphorin signaling pathway (Uchida et al., 2005; Yoshimura et al., 2005). Through this pathway, the C-terminal tail domain of CRMP2 is phosphorylated by cyclin-dependent kinase 5 (CDK5) and glycogen synthase kinase $3 \beta$ (GSK3 $\beta$ ). The phosphorylated CRMP2 negatively regulates microtubule growth and stability, thus having the opposite effect of unphosphorylated CRMP2. Furthermore, hyperphosphorylated CRMP2 has been found at the neurofibrillary tangles in the Alzheimer's disease (AD) brain, suggesting possible involvement in the pathological development of AD (Gu et al., 2000; Lee et al., 2001; Yoshida et al., 1998).

Therefore, the phosphorylation of CRMP2 is fundamental for regulating neuronal polarity as well as the pathological development of the neurodegenerative diseases. The phosphorylation of CRMP2 inhibits the binding between CRMP2 and the microtubule (Lin et al., 2011; Uchida et al., 2005; Yoshimura et al., 2005), albeit its molecular mechanisms are not well understood because of the lack of structural studies of phosphorylated CRMP2. Here we per- 
formed a series of biochemical and structural studies of phospho-mimic CRMP2. Contrary to unphosphorylated CRMP2, phospho-mimic CRMP2 is not able to form a hetero-trimer with GTP-tubulin. Phospho-mimic mutations of the C-terminal tail do not result in major conformational change of the overall structure of CRMP2 but induces small conformational change at the C-terminal tail. Phosphorylation produces a repulsive interaction between CRMP2 and the tubulin/microtubule, thus canceling CRMP2-induced axonal microtubule formation and stabilization.

\section{Materials and Methods}

\section{Preparation of CRMP2 for in vitro experiments}

The CRMP2 constructs CP525 $[7 \times$ His-tag and human CRMP2 residues 1-525] with the S522D mutation (CP525-S522D) and the S522D-T509D-T514D-S518D mutations (CP525-4D) were cloned into pET 21b (Novagen) and pGEX-6p-3 (GE Healthcare) and expressed in Escherichia coli BL21(DE3) cells (Novagen). Constructs were purified and concentrated as previously described (Niwa et al., 2017). Tubulin was purified from porcine brains by six cycles of polymerization and depolymerization as described previously (Morikawa et al., 2015). GTP- $\gamma$ S-tubulin was prepared as described previously (Niwa et al., 2017).

\section{Size exclusion chromatography (SEC) analysis}

$50 \mu \mathrm{l}$ of $250 \mu \mathrm{M}$ protein samples was loaded onto a SEC column (HiLoad 16/60 superdex200 column; GE Healthcare) at a flow rate of $0.5 \mathrm{~mL} \mathrm{~min}{ }^{-1}$ in SEC30 buffer $(20 \mathrm{mM}$ Hepes-KOH $\mathrm{pH}$ 7.4, $30 \mathrm{mM} \mathrm{NaCl}, 1 \mathrm{mM} \mathrm{MgCl}_{2}$ ) at $4^{\circ} \mathrm{C}$ using the ACTA Explorer 10S (GE Healthcare).

\section{Dynamic light scattering analysis}

DLS analysis was performed at $20^{\circ} \mathrm{C}$ using a DynaPro NanoStar (Wyatt technology). Protein samples were diluted to $20 \mu \mathrm{M}$ in dilution buffer (15 mM PIPES $\mathrm{pH} 6.8,1 \mathrm{mM}$ EGTA, $1 \mathrm{mM}$ $\mathrm{MgCl}_{2}$ ) and passed through a $0.22-\mu \mathrm{m}$ PVDF filter before use. The scattering data were fit assuming the scatterers to be globular proteins (Dynamics software; Wyatt Technology).

\section{Crystallization, $X$-ray data collection, and structure determination}

Crystals of CP525-4D, CP525-S522D, and CP525-4D with GSK $3 \beta$ were grown using the vapor diffusion method. Crystallization of CP525-4D and CP525-S522D was performed at $20^{\circ} \mathrm{C}$. That of CP525-4D with GSK3 $\beta$ was performed at $4{ }^{\circ} \mathrm{C}$. Each $2-\mu 1$ protein solution at $8-25 \mathrm{mg} / \mathrm{ml}$ was mixed with $2 \mu \mathrm{l}$ of reservoir buffers (RB). The RB for CP525-4D and CP525-S522D contained $12 \%$ PEG4000, 0.1 M sodium citrate $\mathrm{pH}$ 5.6, and 0.1 M lithium sulfate. The RB for CP525-4D with GSK3 $\beta$ contained $14 \%$ PEG3000, and 0.1 M tri-sodium citrate $\mathrm{pH}$ 5.5. X-ray diffraction data were collected at $-180^{\circ} \mathrm{C}$ at BL26B2 at the SPring-8, Japan (CP525-4D; CP525-S522D) and BLX06DA (CP525-4D with GSK $3 \beta$ ) at the Swiss Light Source, Switzerland. All data were indexed, integrated and processed using an HKL2000 (Otwinowski and Minor, 1997). The structures were solved by molecular replacement using PHASER (McCoy et al., 2007) with the atomic coordinate of wild type CRMP2 (PDB ID: 5X1A) as a starting model (Niwa et al., 2017). Several rounds of iterative model building and refinement were performed using COOT (Emsley and Cowtan, 2004) and PHENIX (Adams et al., 2010). The final crystallographic models of CP525-4D, CP525-S522D, and CP525-4D with GSK3 $\beta$ were solved at $2.0 \AA$, $2.3 \AA$, and 2.8 $\AA$ resolutions with $R_{\text {work }} / R_{\text {free }}$ values of $0.185 / 0.218,0.190 / 0.245$, and $0.202 / 0.266$, respectively. The data collection and refinement statistics are shown in Table I.

\section{In vitro binding assay}

A total of $5 \mu \mathrm{M}$ of GST-GP525s (CP525-wt, CP525-S522D, or CP525-4D) was bound to Glutathione Sepharose 4 FF beads (GE Healthcare) for 2 hours at $4{ }^{\circ} \mathrm{C}$ and washed three times in binding buffer (20 mM Tris- $\mathrm{HCl} \mathrm{pH} 7.4,150 \mathrm{mM} \mathrm{NaCl})$. A total of $5 \mu \mathrm{M}$ of kinases (CDK5-p25 or GSK3 $\beta$ ) in binding buffer $30(20 \mathrm{mM}$ Tris- $\mathrm{HCl} \mathrm{pH} 7.4,30 \mathrm{mM} \mathrm{NaCl}$ ) was then added and incubated for 1 hour at $4^{\circ} \mathrm{C}$, then washed three times. Bound and flow-through fractions were separated by $0.22-\mu \mathrm{m}$ centrifuge tube filter devices.

\section{Results}

\section{Phospho-mimic CRMP2 forms a tetramer but does not form a hetero-trimer with GTP-tubulin}

CRMP2 is primarily phosphorylated at S522 by CDK5, followed by phosphorylation at T509, T514, and S518 by GSK3 $\beta$ (Fig. 1A) (Uchida et al., 2005; Yoshimura et al., 2005). Phosphorylation sites are well conserved between CRMP1 and CRMP2, but not in CRMP3 and CRMP5, indicating their distinct expressions and roles (Bretin et al., 2005; Ponnusamy and Lohkamp, 2013). We thus made two phospho-mimic mutants of CRMP2, CP525-S522D in which S522 was substituted with aspartate, and CP525-4D in which S522, T509, T514, and S518 are all substituted with aspartate (Fig. 1B).

In our previous report, CP525 wild type (CP525-wt) forms a tetramer in solution. The interaction with GTPtubulin ( $\alpha$ - and $\beta$-tubulin hetero-dimer) then breaks the tetramer of CP525-wt into a monomer to form a homogeneous hetero-trimeric complex consisting of the CP525 monomer and GTP-tubulin hetero-dimer (Niwa et al., 2017). Thus, we first examined the interaction between CP525-4D and GTP-tubulin at $4^{\circ} \mathrm{C}$ in vitro by size exclusion chromatography (SEC) to check whether phosphorylation of the 


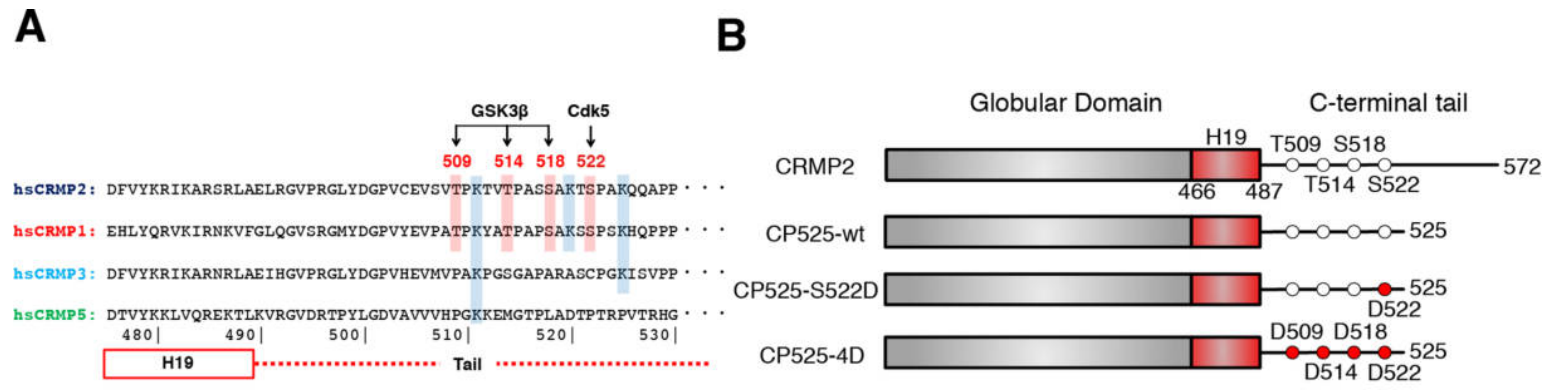

C

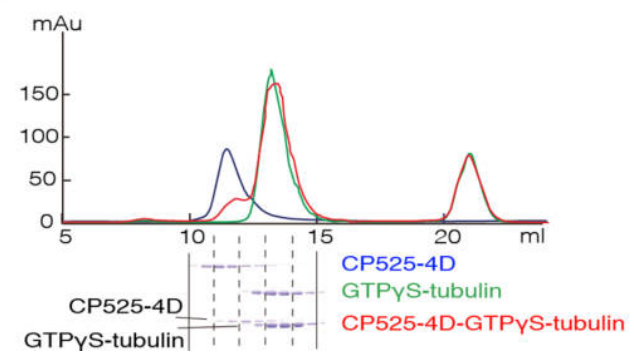

D

\begin{tabular}{|c|c|c|c|c|c|c|}
\hline Molecule & Baseline & sos error & $\mathrm{RH}(\mathrm{nm})$ & Polyd(\%) & MW-R (kDa & ) Intensit \\
\hline CP525-wt & 1.0 & 3.3 & 6.9 & 25.1 & 305.7 & 97.6 \\
\hline CP525-4D & 1.0 & 1.8 & 6.5 & 20.6 & 272.0 & 100.0 \\
\hline GTPYS-Tub & 1.0 & 2.6 & 13.0 & 57.2 & 1416.7 & 98.3 \\
\hline CP525-wt-GTP & 1.0 & 2.2 & 7.7 & 30.4 & 402.7 & 97.8 \\
\hline CP525-4D-GTPgS-Tub & 1.0 & 2.7 & 13.3 & 34.0 & 1438.3 & 96.7 \\
\hline CP525-Wt-GTP & 1.0 & 23.8 & 13.7 & 50.1 & 1608.0 & 56.5 \\
\hline CP525-4D-GTPYS-Tu & 1.0 & 2.3 & 13.8 & 58.3 & 1581.3 & 100.0 \\
\hline
\end{tabular}

Fig. 1. Phospho-mimic CRMP2 does not form a hetero-trimer with GTP-tubulin. (A) Sequence alignment among human CRMPs. Phosphorylation sites at the C-terminal tail are indicated. (B) Schema of three constructs, CP525-wt, CP525-S522D, and CP525-4D. (C) Size exclusion chromatography of CP525-4D (blue), GTP- $\gamma$ S-tubulin (green), and CP525-4D with GTP- $\gamma$ S-tubulin (red), shown with the SDS-PAGE results. (D) DLS of CP525s, GTP- $\gamma$ Stubulin, CP525s with GTP- $\gamma$ S-tubulin, and CP525s with GTP- $\gamma$ S-tubulin-S.

C-terminal tail would abolish the hetero-trimer formation. In this experiment, GTP analog GTP- $\gamma \mathrm{S}$ (guanosine 5'-O$[\gamma$-thio] triphosphate) was used to mimic GTP.

CP525-4D was eluted as the homo-tetramer similar to the CP525-wt (blue chromatogram in Fig. 1C) (Niwa et al., 2017; Stenmark et al., 2007). GTP-tubulin was eluted as the hetero-dimer (green chromatogram in Fig. 1C). When CP525-4D and GTP-tubulin were mixed at a molar rate of $1: 1$, the elution fractions for GTP-tubulin did not change at all, albeit the fraction of CP525-4D slightly shifted to the right (red chromatogram in Fig. 1C). This suggested the possibility that the weak interaction between GTP-tubulin and CP525-4D might mildly slow down the elution speed of CP525-4D.

We further checked the interaction between CP525-4D and GTP-tubulin by dynamic light scattering (DLS) (Fig. 1D). DLS patterns from CP525-wt and CP525-4D both represented low polydispersity and a similar hydrodynamic radius. The estimated molecular weights, if they took globular forms, were 305.7 and $272.0 \mathrm{kDa}$, respectively. These values matched well with the calculated molecular weight of the CP525 tetramer $(270 \mathrm{kDa})$. DLS from GTP$\gamma \mathrm{S}$-tubulin represented a high polydispersity with a large hydrodynamic radius $(\mathrm{RH}=13.0 \mathrm{~nm})$ as reported previously (Niwa et al., 2017). This indicates that oligomerization or aggregation of tubulin occurred in solution. As we reported, a 1:1 mixture of CP525-wt and GTP-tubulin greatly decreased both the hydrodynamic radius $(7.7 \mathrm{~nm})$ and poly- dispersity (30.4\%) (Niwa et al., 2017). Small angle X-ray scattering (SAXS) and size-exclusion chromatography combined with multi-angle light scattering (SEC-MALS) analyses further confirmed that the decreasing hydrodynamic radius reflected the hetero-trimer formation between CP525-wt and GTP-tubulin. However, a 1:1 mixture of CP525-4D and GTP-tubulin showed a much larger hydrodynamic radius $(13.3 \mathrm{~nm}$ ) compared with CP525-wt (Fig. 1D). This indicates that CP525-4D did not form the stable hetero-trimer complex with GTP-tubulin, thus GTP-tubulin could oligomerize in solution.

We further investigated the effect of subtilisin treatment of tubulins for tetrameric formation of CP525-wt and CP525-4D. Limited subtilisin proteolysis of tubulin produces tubulin-S, which lacks the cluster of negatively charged residues found in the C-terminal tails of tubulins (Knipling et al., 1999). Consequently, both the CP525-wt and CP525-4D with GTP-tubulin-S represented the large hydrodynamic radius and large polydispersity in a similar manner, indicating that the C-terminal tails of tubulins are required to form the hetero-trimer between CP525-wt and GTP-tubulin.

\section{Crystal structure of phospho-mimic CRMP2}

We then solved the crystal structure of CP525-4D and CP525-S522D (Table I). As expected from the SEC and DLS results, CP525-4D and CP525-S522D formed a homo- 
Table I. Data COLLECTION AND REFINEMENT STATistics

\begin{tabular}{|c|c|c|c|}
\hline & CP525-4D & CP525-S522D & CP525-4D (GSK3 $\beta)$ \\
\hline Data collection & Spring-8 BL26B2 & Spring-8 BL26B2 & SLS X06DA \\
\hline Wavelength $(\AA)$ & 1.0000 & 1.0000 & 1.0000 \\
\hline Space group & $I 4_{1} 22$ & $I 4,22$ & $I 4,22$ \\
\hline \multicolumn{4}{|l|}{ Cell dimensions } \\
\hline$a, b, c(\AA)$ & $114.1,114.1,196.1$ & $112.4,112.4,196.0$ & $114.3,114.3,194.8$ \\
\hline$\alpha, \beta, \gamma\left(^{\circ}\right)$ & $90.0,90.0,90.0$ & $90.0,90.0,90.0$ & $90.0,90.0,90.0$ \\
\hline Resolution $(\AA)$ & $50-1.80(1.91-1.80)$ & $50-2.30(2.34-2.30)$ & $50-2.80(2.97-2.80)$ \\
\hline$R_{\text {merge }}^{\dagger}$ & $0.055(1.116)$ & $0.083(0.698)$ & $0.819(3.516)$ \\
\hline$R_{\text {meas }}{ }^{\ddagger}$ & $0.059(1.199)$ & $0.089(0.748)$ & $0.835(3.583)$ \\
\hline$C C 1 / 2$ & $99.9(88.3)$ & $99.9(95.0)$ & $98.9(61.0)$ \\
\hline$I / \sigma I$ & $19.8(1.89)$ & $27.5(3.1)$ & $18.0(3.0)$ \\
\hline Completeness (\%) & $99.8(99.8)$ & $99.8(99.0)$ & $99.8(99.0)$ \\
\hline Redundancy & $7.6(7.4)$ & $7.7(7.8)$ & $26.5(27.1)$ \\
\hline \multicolumn{4}{|l|}{ Refinement } \\
\hline Resolution $(\AA)$ & $50-1.8$ & $50-2.3$ & $50-2.8$ \\
\hline No. reflections & 114,502 & 28,103 & 16,296 \\
\hline$R_{\text {work }} / R_{\text {free }}$ & $0.185 / 0.218$ & $0.190 / 0.245$ & $0.202 / 0.266$ \\
\hline \multicolumn{4}{|l|}{ No. atoms } \\
\hline Protein & 3,821 & 3,796 & 3770 \\
\hline $\mathrm{SO}^{4+}$ & 1 & & \\
\hline Water & 431 & 91 & 13 \\
\hline$B$-factors & 31.7 & 40.0 & 49.0 \\
\hline \multicolumn{4}{|l|}{ R.m.s. deviations } \\
\hline Bond lengths $(\AA)$ & 0.007 & 0.008 & 0.010 \\
\hline Bond angles $\left({ }^{\circ}\right)$ & 0.824 & 0.862 & 1.057 \\
\hline
\end{tabular}

* Values in parentheses are for highest-resolution shell.

${ }^{\dagger} R_{\text {merge }}=\Sigma_{h k l} \Sigma_{i i}\left|I_{i}(h k l)-I(h k l)>\right| / \Sigma_{h k l} \Sigma_{i} I_{i}(h k l)$

$* R_{\text {meas }}=R_{\text {r.i.m }}=\Sigma_{h k l}(N / N-1)^{1 / 2} \Sigma_{i} I_{i}(h k l)-<I(h k l)>\mid \Sigma_{h k l} \Sigma_{i} I_{i}(h k l)$

tetramer in crystals (Fig. 2A). Overall conformations of CP525-4D and CP525-S522D were very similar to the previously reported structures of CP525-wt and other CRMPs (Deo et al., 2004; Majava et al., 2008; Niwa et al., 2017; Ponnusamy et al., 2014; Ponnusamy and Lohkamp, 2013; Stenmark et al., 2007). CRMP2 is composed of an Nterminal globular domain - a $\beta$-sheeted upper lobe (M1G68) and an $\alpha / \beta$ barrel lower lobe (G69-R487) - followed by the unstructured C-terminal tail (L488-G572). Cterminal tails extend toward the neighboring molecules and wrap around the globular domain, thus stabilizing tetramer formation. Compared with the homo-tetramer of CP525-wt, the arrangements of the tetramer were slightly modified in CP525-S522D and CP525-4D. The neighboring molecule Mol2 of CP525-S522D moved $\sim 1 \AA$ upward against the molecule Mol1 (Fig. 2B), and that of CP525-4D rotated counterclockwise $\sim 2^{\circ}$ around the molecule Mol1 (Fig. $2 \mathrm{C}$ ). These movements might be driven by the phospho-mimic mutations as detailed below.

In both CP525-4D and CP525-S522D structures, the Cterminal tail was visualized to V506 but the electron densities for the remaining residues were not clearly observed because of the flexibility of the C-terminal tail. Thus, all four phosphorylation sites could not be determined in the atomic structures of CP525-4D and CP525-S522D.

\section{Co-crystallization of phospho-mimic CRMP2 with GSK3及}

To visualize the remaining flexible C-terminal tail, the most realistic way is to stabilize it by forming the complex with some additional factors or proteins. To achieve this, we examined the binding affinity of CP525 with two kinases CDK5 and GSK3 $\beta$. GST-fusion CP525s, GST-CP525-wt, GST-CP525-S522D, and GST-CP525-4D were produced to check the binding to the two kinases. Consequently, the binding between CP525-wt and CDK5 with an activator p25 was not detected at the $5-\mu \mathrm{M}$ concentration (Fig. 3A). However, GSK $3 \beta$ was partially bound to CP525 at the same concentration (Fig. 3B). The binding property of GSK3 $\beta$ to CP525-wt, CP525-S522D, and CP525-4D did not show clear differences. Thus, CP525-4D was cocrystallized with GSK $3 \beta$ at the final concentration of 200 $\mu \mathrm{M}$, to try to visualize the phosphorylated C-terminal.

Crystals of CP525-4D in the presence of the equimolar GSK3 $\beta$ were grown at $4^{\circ} \mathrm{C}$, as described in the Materials and Methods. Unfortunately, however, the electron density corresponding to GSK3 $\beta$ was not observed. The conforma- 
A
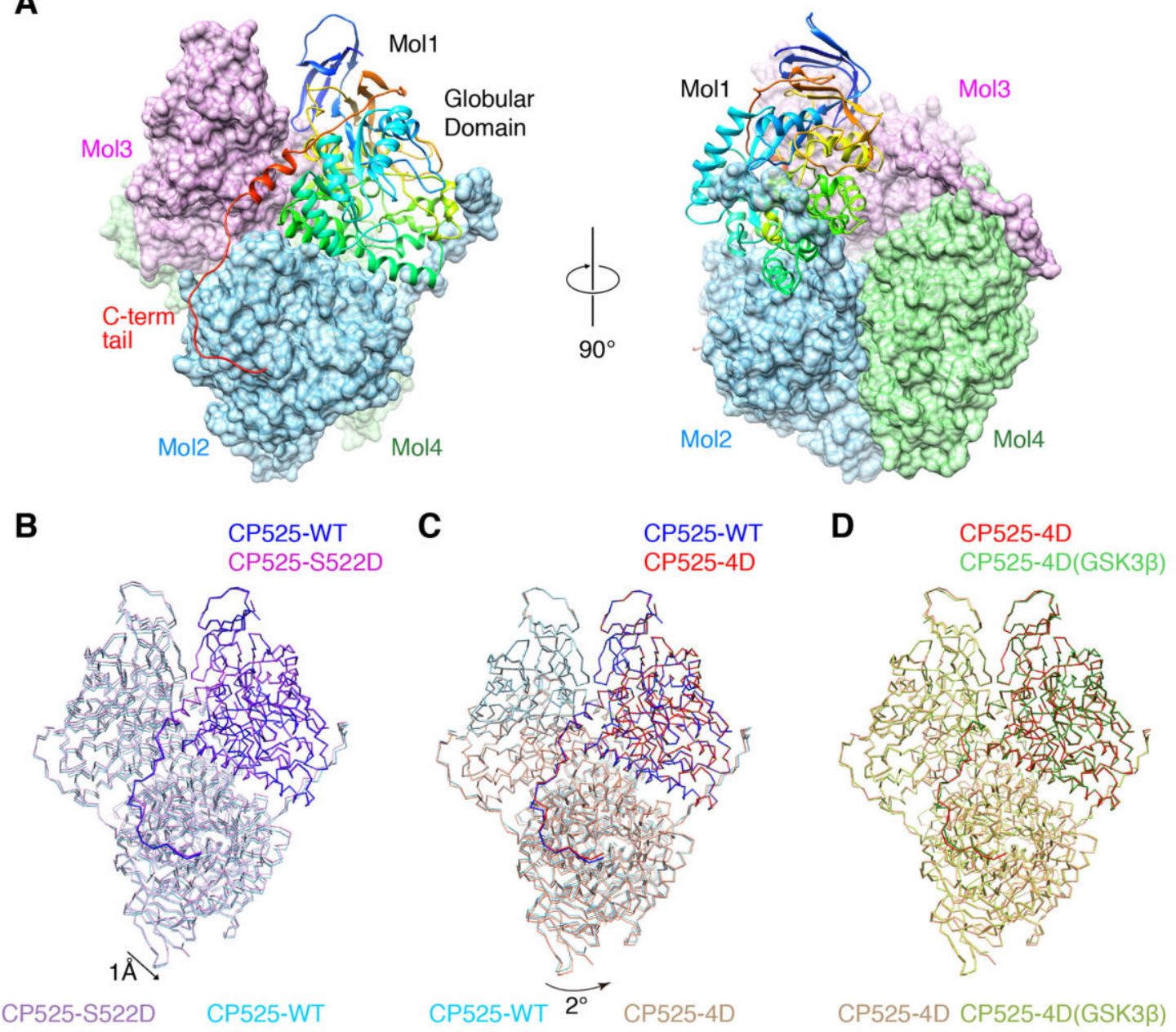

Fig. 2. Crystal structure of phospho-mimic CP525. (A) Crystal structure of CP525-4D homo-tetramer. One monomer is highlighted in rainbow colors: colder colors from the N-terminus and warmer colors to the C-terminus. (B) Comparison of the tetramer arrangements between CP525-wt (blue) and CP525-S522D (purple). (C) Comparison of the tetramer arrangements between CP525-wt (blue) and CP525-4D (red). (D) Comparison of the tetramer arrangements between CP525-4D (red) and CP525-4D in the presence of GSK3 $\beta$ (red).

tion of CP525-4D in the presence of GSK3 $\beta$ was almost same as that in the absence of GSK3 $\beta$ (Fig. 2D).

\section{Structural detail of phospho-mimic CRMP2}

We finally describe here the structural details of phosphomimic mutants of CRMP2. The conformations of CP525S522D and CP525-4D were very similar to CP525-wt (Fig. 2B). Their globular domains took essentially the same structure as that of CP525-wt with low root mean square deviations (RMSDs) (Fig. 4A-B). However, the C-terminal tails of CP525-S522D and CP525-4D took slightly different conformations from that of CP525-wt with relatively high RMSDs (Fig. 4A-B). The C-terminal tail was extended from the last helix H19 of the globular domain. The initial quarter of the C-terminal tail (L488-V506) was visualized in all three structures. The C-terminal tails of CP525-S522D and CP525-4D docked to the neighboring molecule Mol2 and followed the hydrophobic groove formed by the H5-H6 loop, the H10-H11 loop, and the helix H13 (Fig. 4B-C). The small conformational change of the main chain of the C-terminal tail was observed between CP525-wt and the phospho-mimic CP525, albeit the main contacts through the side chains between the Cterminal tail of Moll and the globular domain of Mol2 were mostly conserved (Fig. 4C-D). L491 made first contact surrounded by the four hydrophobic residues of the neighboring Mol2. The salt bridge between R496 of Mol1 and E221 of Mol2 was also observed. The other hydrophobic contacts were also conserved between CP525-wt and the phospho-mimic CP525.

Thus, the phosphorylation at S522, T509, T514, S518 


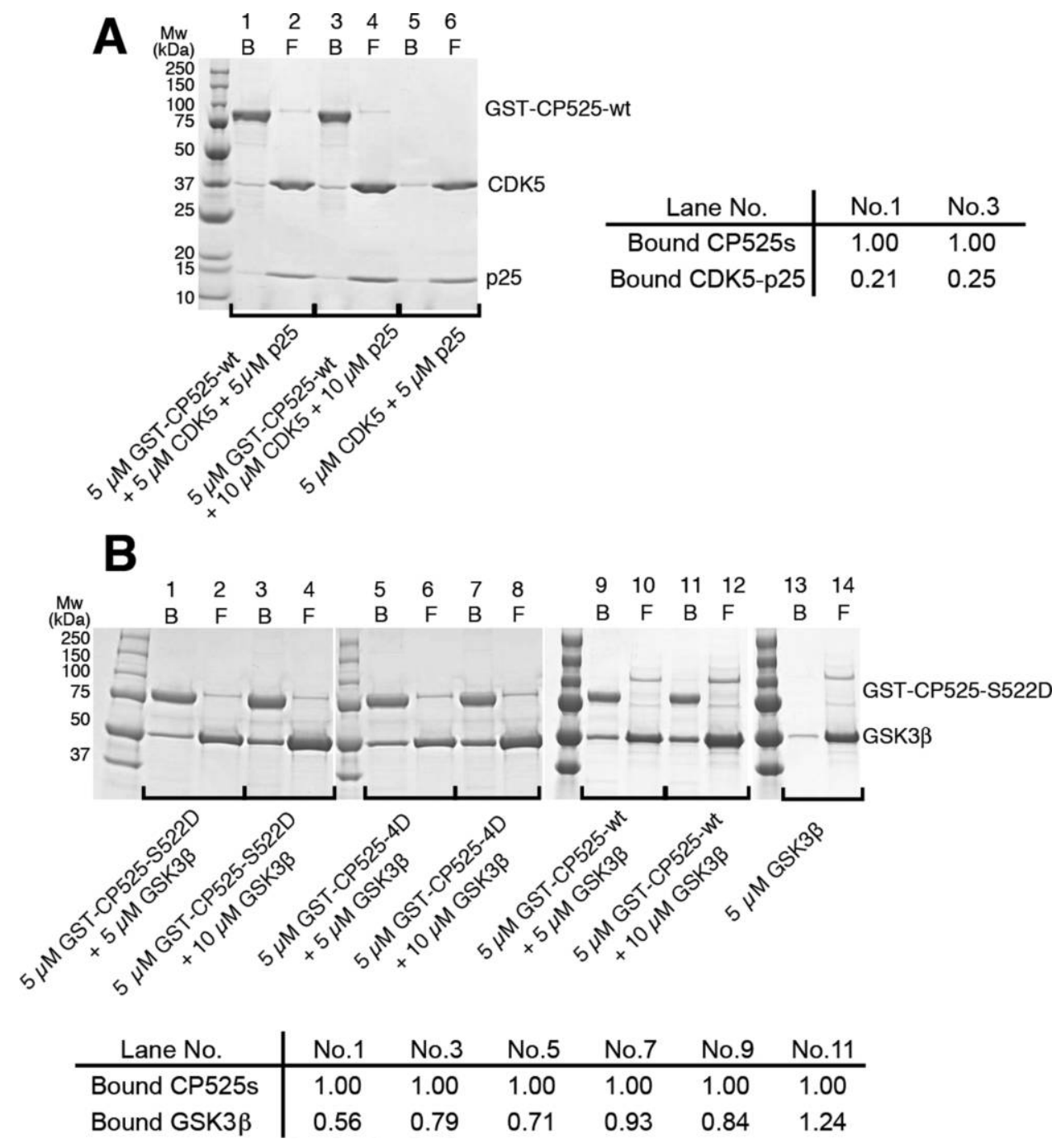

Fig. 3. Binding assay of GST-CP525s (GST-CP525-S522D, GST-CP525-4D, and GST-CP525-wt) with two kinases CDK5-p25 and GSK3ß. B, bound fractions; F, flow-through fractions. (A) Binding assay of $5 \mu \mathrm{M}$ GST-CP525-wt with 5-10 $\mu \mathrm{M}$ CDK5 with $\mathrm{p} 25$. The rate of bound /flow-through fractions of CDK5-p25 did not change with and without GST-CP525-wt. The right table shows the molar rates of CDK5-p25 in the bound fractions if the bound GST-CP525s are set to 1.00 . (B) Binding assays of $5 \mu \mathrm{M}$ GST-CP525s with $5-10 \mu \mathrm{M}$ GSK3 $\beta$. The rate of bound/flow-through fractions of GSK3 $\beta$ increased in the presence of GST-CP525s. The table shows the molar rates of GSK3 $\beta$ in the bound fractions if bound GST-CP525s are set to 1.00 .

did not induce considerable conformational change of the globular domain. However, a $\sim 1 \AA$ translation and $\sim 2^{\circ}$ rotation of Mol2 was observed in the CP525-S522D and CP525-4D crystals (Fig. 2B-C). Considering that the CP525-4D crystals in the presence and absence of GSK3 $\beta$ take the same tetramer arrangements under distinct crystallization conditions (Fig. 2D), the addition of negative charges by phosphorylation at S522 and the other three phosphorylation sites correspond to changing the interaction between the C-terminal tail of Moll and globular domain of Mol2, leading to the conformational change of the initial quarter of the C-terminal tail.

\section{Discussion}

In this study, we performed a series of biochemical and structural analyses of phospho-mimic CRMP2 and clarified the effects of phosphorylation at the C-terminal tail of CRMP2 on interaction with tubulins and microtubules to regulate the microtubule dynamics.

Phospho-mimic CRMP2 forms a homo-tetramer in the absence of tubulin, similar to the wild type CRMP2. Phosphorylation at the C-terminal tail does not change any conformation of the globular domain of CRMP2, but induces a small conformational change of the C-terminal tail. Because the C-terminal tail extends toward the neighboring 
A
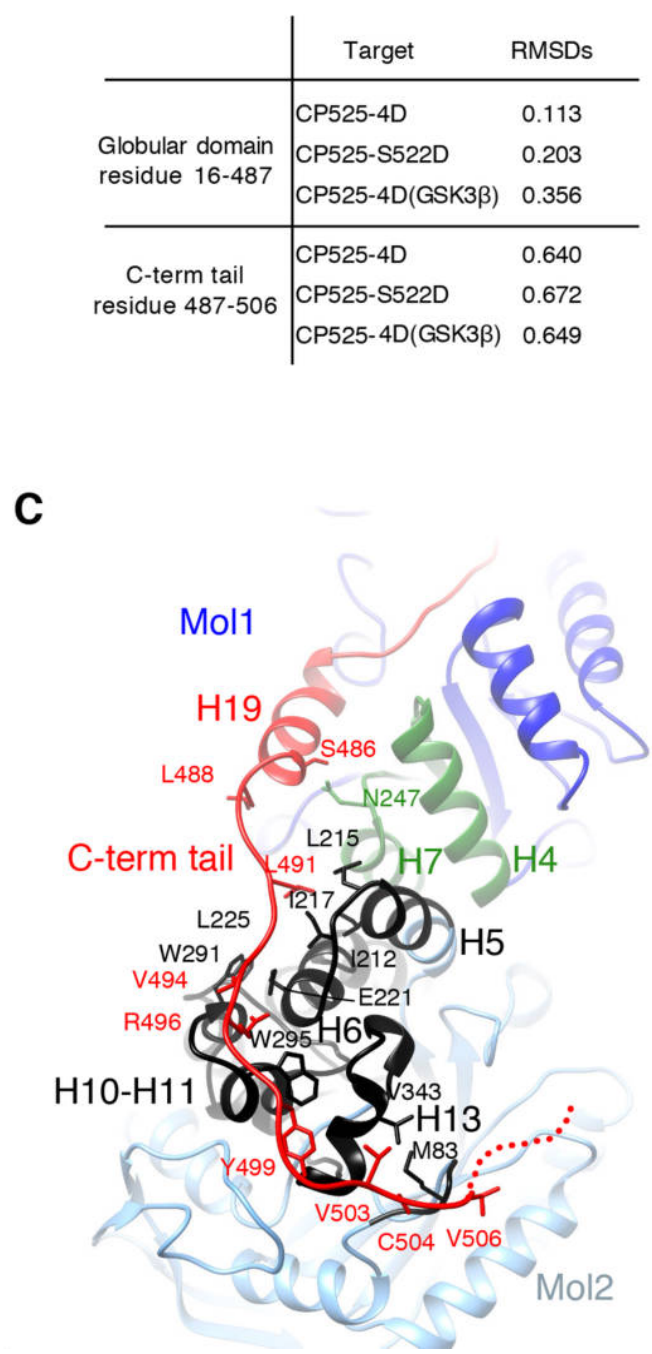

B
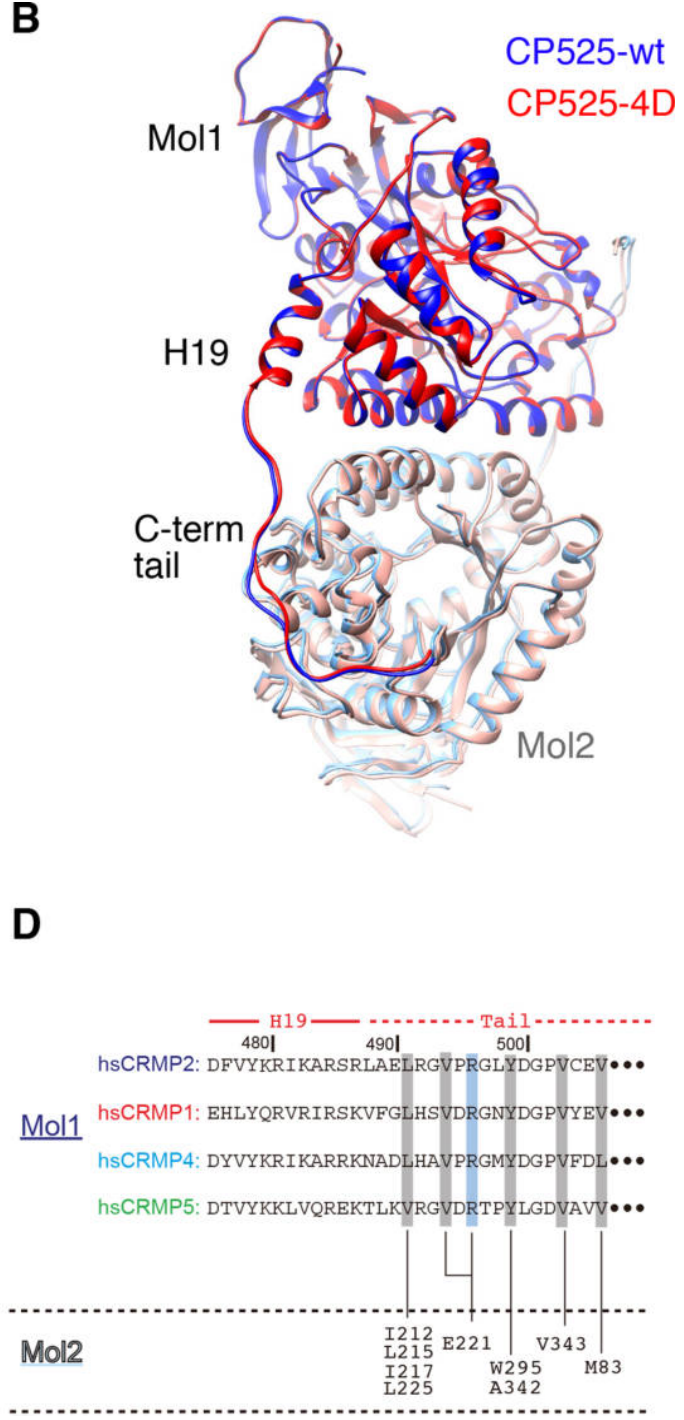

Fig. 4. Structural detail of the C-terminal tail of phopsho-mimic CP525. (A) Root mean square deviations (RMSDs) between CP525-wt and three phospho-mimic CP525s. Residues of the globular domain and the C-terminal tail are separately calculated. (B) Comparison between CP525-wt (blue) and CP525-4D (red) structures. (C) Structural detail of the C-terminal tail of CP525-4D. (D) Sequence alignment of the helix H19 and the C-terminal tail. The interacting residues between the C-terminal tail of Mol1 and the globular domain of Mol2 are shown. Basic residues, blue background; hydrophobic residues, gray background.

monomer to support the homo-tetramer formation of CRMP2, the addition of negative charges by phosphorylation changes the interaction between the C-terminal tail and the neighboring globular domain, leading to the small change of the conformation of C-terminal tail as well as a slight rearrangement of monomer-monomer interaction in the tetramer.

Our biochemical studies further suggested that the phosphorylation of the C-terminal tail interferes with homogeneous hetero-trimer formation between the CRMP2 monomer and the GTP-tubulin hetero-dimer. It is also suggested that deletion of the E-hook of tubulin inhibits the hetero-trimer formation even for unphosphorylated CRMP2. Amino acid sequence alignments show that three lysine residues conserved between CRMP1 and CRMP2 are present around the phosphorylation area of the $\mathrm{C}$ terminal tail (Fig. 1A). Especially, S522 is surrounded by two lysine residues. However, the E-hook of tubulin is rich in acidic residues. Thus, the ionic interaction between the basic C-terminal tail and the acidic E-hook serve as the priming interaction between CRMP2 and GTP-tubulin, which is followed by stable contact by their globular domains to elongate the axonal microtubules (Niwa et al., 2017). Thus, the phosphorylation of the C-terminal tail, which adds the 
negative charged side chains between the lysine residues, produces repulsion between the phosphorylated C-terminal tail and the E-hook, thus interfering with their interactions.

As previously reported, phosphorylation of the Cterminal tail also inhibits interaction between CRMP2 and the microtubule (Lin et al., 2011; Uchida et al., 2005; Yoshimura et al., 2005). Thus, phosphorylation of the C-terminal tail could inhibit interactions both with tubulindimers and with microtubules. In other words, phosphorylation of the C-terminal tail could negatively regulate the CRMP2-induced axonal microtubule formation as well as the CRMP2-led stabilization of microtubules. Hence, the structural information of the phosphorylated $\mathrm{C}$-terminal tail that we attempted to visualize is the key to understand the molecular mechanisms through which CRMP2 controls the microtubule dynamics. Although the association between CP525 and GSK3 $\beta$ was clearly detected, we could not visualize the density corresponding to the C-terminal tail of CRMP2 even in the presence of GSK3 $\beta$. One possible reason might be that flexibility of the C-terminal tail could not be suppressed by the GSK3 $\beta$ binding. The dissociation of GSK3 $\beta$ from CP525 in the crystal also might be possible. The enhancement of binding between them by chemical cross-linking might help us to overcome this issue. We should also try the longer CRMP2 construct to make the stable association or phosphorylated CRMP2 instead of the phospho-mimic CRMP2 mutant. The phospho-regulation might serve as the structural basis for how semaphorininduced phosphorylation of CRMP2 switches the CRMP2 function from axon elongation through effective axonal microtubule formation to axon repulsion through the destabilization of microtubules. Future structural studies to visualize the full length of CRMP2 including the C-terminal tail are expected.

Acknowledgments. We thank Drs. Vincent Olieric and Takashi Tomizaki at SLS and Dr. Go Ueno and Mr. Yuki Nakamura at SPring-8 for their technical support. This work was supported by JSPS KAKENHI (No. 15K08168 to R.N.), the Takeda Science Foundation to R.N. and the Mochida Memorial Foundation for Medical and Pharmaceutical Research to R.N.

\section{Competing interests}

The authors declare that they have no conflict of interest.

\section{Data and materials availability}

The coordinates for the crystal structures have been deposited in the Protein Data Bank under following accession codes. CP525-4D, 5YZ5; CP525-S522D, 5YZA; CP525-4D (GSK3 $\beta$ ), 5YZB. Correspondence and requests for materials should be addressed to R.N. (ryonitta@ med.kobe-u.ac.jp).

\section{References}

Adams, P.D., Afonine, P.V., Bunkoczi, G., Chen, V.B., Davis, I.W., Echols, N., Headd, J.J., Hung, L.W., Kapral, G.J., Grosse-Kunstleve, R.W., McCoy, A.J., Moriarty, N.W., Oeffner, R., Read, R.J., Richardson, D.C., Richardson, J.S., Terwilliger, T.C., and Zwart, P.H. 2010. PHENIX: a comprehensive Python-based system for macromolecular structure solution. Acta Crystallogr. D, 66: 213-221.

Arimura, N., Menager, C., Fukata, Y., and Kaibuchi, K. 2004. Role of CRMP-2 in neuronal polarity. J. Neurobiol., 58: 34-47.

Arimura, N. and Kaibuchi, K. 2007. Neuronal polarity: from extracellular signals to intracellular mechanisms. Nat. Rev. Neurosci., 8: 194-205.

Bretin, S., Reibel, S., Charrier, E., Maus-Moatti, M., Auvergnon, N., Thevenoux, A., Glowinski, J., Rogemond, V., Premont, J., Honnorat, J., and Gauchy, C. 2005. Differential expression of CRMP1, CRMP2A, CRMP2B, and CRMP5 in axons or dendrites of distinct neurons in the mouse brain. J. Comp. Neurol., 486: 1-17.

Deo, R.C., Schmidt, E.F., Elhabazi, A., Togashi, H., Burley, S.K., and Strittmatter, S.M. 2004. Structural bases for CRMP function in plexindependent semaphorin3A signaling. EMBO J., 23: 9-22.

Emsley, P. and Cowtan, K. 2004. Coot: model-building tools for molecular graphics. Acta Crystallogr. D, 60: 2126-2132.

Fukata, Y., Itoh, T.J., Kimura, T., Menager, C., Nishimura, T., Shiromizu, T., Watanabe, H., Inagaki, N., Iwamatsu, A., Hotani, H., and Kaibuchi, K. 2002a. CRMP-2 binds to tubulin heterodimers to promote microtubule assembly. Nat. Cell Biol., 4: 583-591.

Fukata, Y., Kimura, T., and Kaibuchi, K. 2002b. Axon specification in hippocampal neurons. Neurosci. Res., 43: 305-315.

Goshima, Y., Nakamura, F., Strittmatter, P., and Strittmatter, S.M. 1995. Collapsin-induced growth cone collapse mediated by an intracellular protein related to UNC-33. Nature, 376: 509-514.

Gu, Y., Hamajima, N., and Ihara, Y. 2000. Neurofibrillary tangleassociated collapsin response mediator protein-2 (CRMP-2) is highly phosphorylated on Thr-509, Ser-518, and Ser-522. Biochemistry, 39: $4267-4275$.

Inagaki, N., Chihara, K., Arimura, N., Menager, C., Kawano, Y., Matsuo, N., Nishimura, T., Amano, M., and Kaibuchi, K. 2001. CRMP-2 induces axons in cultured hippocampal neurons. Nat. Neurosci., 4: 781-782.

Knipling, L., Hwang, J., and Wolff, J. 1999. Preparation and properties of pure tubulin S. Cell Motil. Cytoskeleton, 43: 63-71.

Lee, V.M., Goedert, M., and Trojanowski, J.Q. 2001. Neurodegenerative tauopathies. Annu. Rev. Neurosci., 24: 1121-1159.

Li, W., Herman, R.K., and Shaw, J.E. 1992. Analysis of the Caenorhabditis elegans axonal guidance and outgrowth gene unc-33. Genetics, 132: 675-689.

Lin, P.C., Chan, P.M., Hall, C., and Manser, E. 2011. Collapsin response mediator proteins (CRMPs) are a new class of microtubule-associated protein (MAP) that selectively interacts with assembled microtubules via a taxol-sensitive binding interaction. J. Biol. Chem., 286: 4146641478.

Majava, V., Loytynoja, N., Chen, W.Q., Lubec, G., and Kursula, P. 2008. Crystal and solution structure, stability and post-translational modifications of collapsin response mediator protein 2. FEBS J., 275: 45834596.

Maniar, T.A., Kaplan, M., Wang, G.J., Shen, K., Wei, L., Shaw, J.E., Koushika, S.P., and Bargmann, C.I. 2012. UNC-33 (CRMP) and ankyrin organize microtubules and localize kinesin to polarize axondendrite sorting. Nat. Neurosci., 15: 48-56.

McCoy, A.J., Grosse-Kunstleve, R.W., Adams, P.D., Winn, M.D., Storoni, L.C., and Read, R.J. 2007. Phaser crystallographic software. J. Appl. Crystallogr., 40: 658-674.

Morikawa, M., Yajima, H., Nitta, R., Inoue, S., Ogura, T., Sato, C., and Hirokawa, N. 2015. X-ray and Cryo-EM structures reveal mutual conformational changes of Kinesin and GTP-state microtubules upon bind- 
ing. EMBO J., 34: 1270-1286.

Nakata, T., Niwa, S., Okada, Y., Perez, F., and Hirokawa, N. 2011. Preferential binding of a kinesin-1 motor to GTP-tubulin-rich microtubules underlies polarized vesicle transport. J. Cell Biol., 194: 245-255.

Niwa, S., Nakamura, F., Tomabechi, Y., Aoki, M., Shigematsu, H., Matsumoto, T., Yamagata, A., Fukai, S., Hirokawa, N., Goshima, Y., Shirouzu, M., and Nitta, R. 2017. Structural basis for CRMP2-induced axonal microtubule formation. Sci. Rep., 7: 10681.

Otwinowski, Z. and Minor, W. 1997. Processing of X-ray diffraction data collected in oscillation mode. Method Enzymol., 276: 307-326.

Ponnusamy, R. and Lohkamp, B. 2013. Insights into the oligomerization of CRMPs: crystal structure of human collapsin response mediator protein 5. J. Neurochem., 125: 855-868.

Ponnusamy, R., Lebedev, A.A., Pahlow, S., and Lohkamp, B. 2014. Crystal structure of human CRMP-4: correction of intensities for latticetranslocation disorder. Acta Crystallogr. D, 70: 1680-1694.

Stenmark, P., Ogg, D., Flodin, S., Flores, A., Kotenyova, T., Nyman, T., Nordlund, P., and Kursula, P. 2007. The structure of human collapsin response mediator protein 2, a regulator of axonal growth. J. Neurochem., 101: 906-917.

Uchida, Y., Ohshima, T., Sasaki, Y., Suzuki, H., Yanai, S., Yamashita, N., Nakamura, F., Takei, K., Ihara, Y., Mikoshiba, K., Kolattukudy, P., Honnorat, J., and Goshima, Y. 2005. Semaphorin3A signalling is mediated via sequential Cdk5 and GSK3 $\beta$ phosphorylation of CRMP2: implication of common phosphorylating mechanism underlying axon guidance and Alzheimer's disease. Genes Cells: Devoted to Molecular \& Cellular Mechanisms, 10: 165-179.

Yoshida, H., Watanabe, A., and Ihara, Y. 1998. Collapsin response mediator protein-2 is associated with neurofibrillary tangles in Alzheimer's disease. J. Biol. Chem., 273: 9761-9768.

Yoshimura, T., Kawano, Y., Arimura, N., Kawabata, S., Kikuchi, A., and Kaibuchi, K. 2005. GSK-3 $\beta$ regulates phosphorylation of CRMP-2 and neuronal polarity. Cell, 120: 137-149.

(Received for publication, December 11, 2017, accepted, December 29, 2017 and published online, February 23, 2018) 